\title{
Patterns of multimorbidity and risk of disability in community-dwelling older persons
}

\author{
Alessandra Marengoni ${ }^{1,2}$ (C) Roselyne Akugizibwe ${ }^{2} \cdot$ Davide L. Vetrano $^{2,3} \cdot$ Albert Roso-Llorach $^{4,5} \cdot$ Graziano Onder $^{6}$. \\ Anna-Karin Welmer ${ }^{2,7,8,9} \cdot$ Amaia Calderón-Larrañaga $^{2}$
}

Received: 25 October 2020 / Accepted: 3 December 2020 / Published online: 13 February 2021

(c) The Author(s) 2021

\begin{abstract}
The aim was to analyze the association between specific patterns of multimorbidity and risk of disability in older persons. Data were gathered from the Swedish National Study on Aging and Care in Kungsholmen (SNAC-K); 2066 60 + year-old participants living in the community and free from disability at baseline were grouped according to their multimorbidity patterns and followed-up for six years. The association between multimorbidity patterns and disability in basic (ADL) and instrumental (IADL) activities of daily living was examined through multinomial models. Throughout the follow-up, 434 (21.0\%) participants developed at least one ADL and $310(15.0 \%)$ at least one IADL. Compared to the unspecific pattern, which included diseases not exceeding their expected prevalence in the total sample, belonging to the cardiovascular/anemia/dementia, the sensory impairment/cancer and the musculoskeletal/respiratory/gastrointestinal patterns was associated with a higher risk of developing both ADL and IADL, whereas subjects in the metabolic/sleep disorders pattern showed a higher risk of developing only IADL. Multimorbidity patterns are differentially associated with incident disability, which is important for the design of future prevention strategies aimed at delaying functional impairment in old age, and for a better healthcare resource planning.
\end{abstract}

Keywords Multimorbidity patterns $\cdot$ Disability $\cdot$ Older persons

\section{Introduction}

The co-occurrence of multiple diseases in the same individual, i.e., multimorbidity, is the most prevalent and burdensome condition in older adults [1], accounting for many

Alessandra Marengoni and Roselyne Akugizibwe have contributed equally to this work.

Alessandra Marengoni

Alessandra.marengoni@unibs.it

1 Department of Clinical and Experimental Sciences, University of Brescia, Brescia, Italy

2 Aging Research Center, Department of Neurobiology, Care Sciences and Society, Karolinska Institutet and Stockholm University, Stockholm, Sweden

3 Centro Medicina Dell'Invecchiamento, Fondazione Policlinico Universitario “A. Gemelli” IRCCS, Università Cattolica del Sacro Cuore, Rome, Italy

4 Fundació Institut Universitari per a la Recerca a l'Atenció Primària de Salut Jordi Gol i Gurina (IDIAPJGol), Gran Via Corts Catalanes 587, Barcelona, Spain years of life lived with disability [2] and functional decline over time $[3,4]$. Despite its common use in research, assessing the impact of simple counts of chronic diseases on health outcomes may be insufficient to explain the heterogeneity across older persons' functional status. In the past few years, patterns of systematically clustering diseases have been increasingly investigated, in an attempt to explain

5 Universitat Autònoma de Barcelona, Campus de la UAB, Bellaterra (Cerdanyola del Vallès), Spain

6 Department of Cardiovascular, Endocrine-Metabolic Diseases and Aging, Istituto Superiore Di Sanità, Rome, Italy

7 Division of Physiotherapy, Department of Neurobiology, Care Sciences and Society, Karolinska Institutet, Stockholm, Sweden

8 Karolinska University Hospital, Stockholm, Sweden

9 Stockholm Gerontology Research Center, Stockholm, Sweden 
their potential synergistic effects on several health outcomes $[5,6]$. In previous reports from our group, we showed that persons affected by specific multimorbidity patterns have increased risk of death [7] and dementia onset [6], compared to those belonging to an unspecific pattern.

Similarly, persons affected by specific patterns of multimorbidity may be expected to differ in their risk of developing functional decline and disability over time, but only few studies have evaluated the association between multimorbidity patterns and disability, mostly at a cross-sectional level [5]. In the Health and Retirement Study, subjects within a pattern comprising arthritis, hypertension and depression showed the highest level of limitations in basic and instrumental activities of daily living compared to healthy participants [8]. In another study, the combination of somatic and mental diseases was associated with substantially greater prospective disability than combinations comprised exclusively of somatic conditions [9].

The aim of this study was to analyze the association between specific patterns of multimorbidity and the sixyear risk of disability in community-dwelling Swedish older persons.

\section{Methods}

\section{Study population}

We carried out a prospective cohort study using data from the Swedish National study on Aging and Care in Kungsholmen (SNAC-K). SNAC-K is an ongoing longitudinal study including people aged $60+$ years (range: 60-104) living either in the community or in institutions in the Kungsholmen central area of Stockholm, Sweden [10]. Participants were randomly selected from 11 age cohorts $(60,66,72,78$, $81,84,87,90,93,96$ and $99+$ years) during the baseline wave (2001-2004), leading to a study population of 3363 individuals (response rate: 73.3\%). Follow-up interviews are performed every 3 years for individual 78 years and above and every 6 years for individuals younger than 78 years.

The present study included data from 2741 individuals with multimorbidity (i.e., $2+$ chronic diseases) and living in the community at baseline, who were followed-up for 6 years. We excluded: (1) individuals with any limitations in basic or instrumental activities of daily living (i.e., ADL and IADL) at baseline ( $n=592)$, (2) subjects receiving formal home care at baseline $(n=51)$, and (3) participants with no data for ADL or IADL at follow-up $(n=32)$. The analytical sample consisted of 2066 participants.

Ethical approval was obtained from the Regional Ethics Review Board in Stockholm County, and informed consent was obtained from participants or a proxy (if the participant had severe cognitive impairment) before participation in the study.

\section{Chronic disease assessment}

This was done using participants' medical history, clinical examination, inpatient and outpatient registers, self-reported information and proxy interviews. In addition, laboratory tests and medications were used for the diagnosis of specific conditions. Chronic diseases were coded according to the International Classification of Diseases, 10th revision (ICD10) and were grouped into 60 categories based on an operationalization that has been developed and applied to SNAC-K data in a previous proof-of-concept study (1). The clinical criteria for grouping the identified diagnoses included similarities in their pathophysiology, treatment, prognosis and prevalence.

\section{Disability assessment}

The primary outcomes were incident disability in at least one ADL (i.e., bathing, dressing, toilet use, urinary continence, transferring self from bed and eating) and IADL (i.e., phone use, grocery shopping, food preparation, housekeeping, laundry, use of public transport, handling finances and medication use). These were assessed using a questionnaire administered by a qualified nurse, and each item was graded as "independent" or "dependent."

\section{Covariates}

Data on age, sex, education, marital status, living arrangement, informal and formal care were gathered through questionnaires. Education was measured as the highest attained level. Formal social care includes help with household chores and personal care. It is financed by the municipality and may be delivered either by the municipality or a private company that has a contract with the municipality. Informal social care covers service or care assistance from relatives, friends, neighbors, or volunteer/non-profit organizations. The amount of formal and informal care a person received was recorded as hours per month.

\section{Analysis}

We employed a fuzzy c-means cluster analysis to classify the study participants into six homogeneous patterns of multimorbidity: psychiatric diseases; cardiovascular diseases, anemia and dementia; metabolic and sleep disorders; sensory impairments and cancer; musculoskeletal, respiratory and gastrointestinal diseases; and unspecific (Table S1). Participants in the unspecific pattern had 
Table 1 Baseline characteristics of participants by the different outcomes

\begin{tabular}{|c|c|c|c|c|c|c|c|}
\hline Characteristics & Total $N=2066$ & $\begin{array}{l}\text { ADL disability } \\
n=434\end{array}$ & $\begin{array}{l}\text { No ADL disability } \\
n=1,027\end{array}$ & $P$-value & $\begin{array}{l}\text { IADL disability } \\
n=310\end{array}$ & $\begin{array}{l}\text { No IADL disability } \\
n=1,205\end{array}$ & $P$-value \\
\hline Age, $n(\%)$ & & & & $<0.001$ & & & $<0.001$ \\
\hline$<78$ years & $1,267(61.3)$ & $254(58.5)$ & 747 (72.7) & & $64(20.6)$ & $937(77.8)$ & \\
\hline$\geq 78$ years & 799 (38.7) & $180(41.5)$ & $280(27.3)$ & & $246(79.4)$ & $268(22.2)$ & \\
\hline Gender, $n(\%)$ & & & & $<0.001$ & & & $<0.003$ \\
\hline Males & $768(37.2)$ & $84(19.4)$ & $457(44.5)$ & & $92(29.7)$ & $464(38.5)$ & \\
\hline Females & $1,298(62.8)$ & $350(80.6)$ & $570(55.5)$ & & $218(70.3)$ & $741(61.5)$ & \\
\hline Education, $n(\%)$ & & & & $<0.001$ & & & $<0.001$ \\
\hline Elementary & $294(14.3)$ & $63(14.5)$ & $112(10.9)$ & & $68(22.1)$ & $122(10.1)$ & \\
\hline High school & $1,036(50.3)$ & $223(51.5)$ & $491(47.9)$ & & $166(53.9)$ & $580(48.1)$ & \\
\hline University & $728(35.4)$ & 147 (33.9) & $423(41.2)$ & & $74(24.0)$ & $503(41.7)$ & \\
\hline Marital status, $n(\%)$ & & & & $<0.001$ & & & $<0.001$ \\
\hline Unmarried & $340(16.5)$ & $72(16.6)$ & $173(16.9)$ & & $40(12.9)$ & $210(17.4)$ & \\
\hline Married & $970(47.2)$ & $188(43.3)$ & $544(53.0)$ & & $114(36.8)$ & $635(52.7)$ & \\
\hline Divorced & $302(14.7)$ & $70(16.1)$ & $149(14.5)$ & & $41(13.2)$ & $182(15.1)$ & \\
\hline Widow & 445 (21.6) & $104(24.0)$ & $160(15.6)$ & & $115(37.1)$ & $177(14.7)$ & \\
\hline Polypharmacy, $n(\%)$ & & & & $<0.001$ & & & $<0.001$ \\
\hline$\geq 5$ drugs & $701(33.9)$ & $185(42.6)$ & $269(26.2)$ & & $157(50.6)$ & $324(26.9)$ & \\
\hline$\geq 10$ drugs & $103(5.0)$ & $27(6.2)$ & $31(3.0)$ & & $31(10.0)$ & $33(2.7)$ & \\
\hline Chronic diseases, $n(\%)$ & & & & $<0.001$ & & & $<0.001$ \\
\hline$\geq 3$ chronic diseases & $1,558(75.4)$ & $355(81.8)$ & $713(69.4)$ & & $276(89.0)$ & $845(70.1)$ & \\
\hline$\geq 4$ chronic diseases & $1,046(50.6)$ & $254(58.5)$ & $452(44.0)$ & & $228(73.5)$ & $524(43.5)$ & \\
\hline $\begin{array}{l}\text { Multimorbidity patterns, } \\
n(\%)\end{array}$ & & & & $<0.001$ & & & $<0.001$ \\
\hline Psychiatric & $109(5.3)$ & $27(6.2)$ & $51(5.0)$ & & $15(4.8)$ & $65(5.4)$ & \\
\hline Cardio/Anemia/Dementia & $96(4.6)$ & $18(4.1)$ & $24(2.3)$ & & $26(8.4)$ & $24(2.0)$ & \\
\hline Metabolic/Sleep & $259(12.5)$ & $47(10.8)$ & $116(11.3)$ & & 37 (11.9) & $135(11.2)$ & \\
\hline Sensory/Cancer & $178(8.6)$ & $47(10.8)$ & $71(6.9)$ & & $56(18.1)$ & $72(6.0)$ & \\
\hline MSK/Resp/GI & $320(15.5)$ & $83(19.1)$ & $143(13.9)$ & & $65(21.0)$ & $170(14.1)$ & \\
\hline Unspecific & $1,104(53.4)$ & $212(48.8)$ & $622(60.6)$ & & $111(35.8)$ & $739(61.3)$ & \\
\hline
\end{tabular}

Test: Pearson's chi-squared

Missing data: education 8, marital status 9, informal care 33

Abbreviations: GI gastro-intestinal diseases, Resp respiratory diseases, MSK musculoskeletal, Cardio cardiovascular diseases

diseases that did not specifically characterize this pattern [11].

We used multinomial logistic regression models to test the association between multimorbidity patterns and the different outcomes, and results were reported as relative rate ratios (RRR) with their confidence intervals. Secondary outcomes were: (1) death during follow-up, which was derived from the Swedish death register, and (2) loss to follow-up. Potential interactions between multimorbidity patterns and sex were assessed, and sex-stratified analyses were carried out subsequently. Analyses were carried out using Stata IC/15.0, and a $P$-value of $<0.05$ was considered statistically significant.

\section{Results}

This study consisted of $1267(61.3 \%)$ participants aged $<78$ years and $799(38.7 \%)$ participants aged $\geq 78$ years (Table 1). Most of the participants in the study were female $(n=1298,62.8 \%)$. Participants were distributed across the six different multimorbidity patterns as follows: psychiatric (5.3\%), cardiovascular/anemia/dementia (4.6\%), metabolic/sleep disorders (12.5\%), sensory impairment/cancer (8.6\%), musculoskeletal/respiratory/gastrointestinal (15.5\%) and an unspecific pattern (53.4\%) including diseases not exceeding their expected 
prevalence in the total sample. By the end of the 6-year follow-up, $434(21.0 \%)$ of the participants developed at least one ADL. Within this group of subjects, 58.5\% were $<78$ years, the majority were female $(80.6 \%)$ and most had $\geq 4$ chronic conditions (58.5\%). During the same period, 310 (15.0\%) participants developed at least one IADL; they were mostly aged $\geq 78$ years $(79.3 \%)$ and females $(70.3 \%), 51.84 \%$ had polypharmacy and most had $\geq 4$ chronic conditions (73.6\%).

Belonging to the cardiovascular/anemia/dementia (RRR $2.09,95 \%$ CI $1.08,4.06)$, the sensory impairment/cancer (RRR 1.62, 95\% CI 1.05, 2.48) and the musculoskeletal/respiratory/gastrointestinal (RRR 1.40, 95\% CI 1.02, 1.94) patterns was significantly associated with a higher risk of developing ADL, compared to the unspecific pattern (Table 2). Moreover, subjects in the cardiovascular/anemia/dementia pattern (RRR 3.11, 95\% CI 1.64, 5.89), the sensory impairment/cancer pattern (RRR 2.11, 95\% CI 1.36, 3.28), the musculoskeletal/respiratory/gastrointestinal pattern (RRR $1.97,95 \%$ CI 1.34, 2.90), and those in the metabolic/sleep disorders pattern (RRR 1.58, 95\% CI 1.00, 2.48) showed a higher risk of developing IADL compared with those in the unspecific pattern (Table 2).

There was no statistically significant interaction between the multimorbidity patterns and sex. Sex stratification strengthened the association between the cardiovascular/ anemia/dementia pattern and the risk of developing $\mathrm{ADL}$ among females (RRR 3.42, 95\% CI 1.42, 8.22), while the association with other patterns was weakened and

Table 2 Association (RRR and 95\%CI) of multimorbidity patterns with the risk of developing ADL and IADL

\begin{tabular}{lll}
\hline Multimorbidity patterns & Cases/at risk & RRR $(95 \% \mathrm{CI})$ \\
\hline$A D L$ & & \\
Unspecific & $212 / 1104$ & Ref. \\
Psychiatric & $27 / 109$ & $1.50(0.91,2.49)$ \\
Cardio/Anemia/Dementia & $18 / 96$ & $2.09(1.08,4.06)^{*}$ \\
Metabolic/Sleep & $47 / 259$ & $1.41(0.95,2.07)$ \\
Sensory/Cancer & $47 / 178$ & $1.62(1.05,2.48)^{*}$ \\
MSK/Resp/GI & $83 / 320$ & $1.40(1.02,1.94)^{*}$ \\
IADL & & \\
Unspecific & $111 / 1104$ & Ref. \\
Psychiatric & $15 / 109$ & $1.79(0.93,3.43)$ \\
Cardio/Anemia/Dementia & $26 / 96$ & $3.11(1.64,5.89)^{* * *}$ \\
Metabolic/Sleep & $37 / 259$ & $1.58(1.00,2.48)^{*}$ \\
Sensory/Cancer & $56 / 178$ & $2.11(1.36,3.28)^{* * *}$ \\
MSK/Resp/GI & $65 / 320$ & $1.97(1.34,2.90)^{* * *}$ \\
\hline
\end{tabular}

Model adjusted by age, sex, education level, marital status

Abbreviations: RRR relative rate ratio, GI gastro-intestinal diseases, Resp respiratory diseases, MSK musculoskeletal, Cardio cardiovascular diseases, $C I$ confidence interval

$P$-values: $*<0.05, * * \leq 0.01, * * * \leq 0.001$ non-significant. Among males, the risk of developing ADL was strengthened for the sensory impairment/cancer pattern (RRR 2.42, 95\% CI 1.14, 5.15), but no significant associations were observed with the other patterns. For IADL, with the exception of the metabolic/sleep disorder pattern, the associations were strengthened for all the patterns among females, including the psychiatric pattern, which became statistically significant (RRR $2.20,95 \%$ CI 1.02, 4.73). On the contrary, the associations with all patterns were attenuated and became non-significant among males (Table S2).

The association between the multimorbidity patterns and death or dropout can be found as supplementary material (Table S3).

\section{Discussion}

Compared to the unspecific pattern, belonging to the cardiovascular/anemia/dementia pattern, the sensory impairment/cancer pattern and the musculoskeletal/respiratory/ gastrointestinal pattern was significantly associated with a higher risk of developing both ADL and IADL, whereas subjects in the metabolic/sleep disorders pattern showed a higher risk of developing only IADL. Furthermore, in stratified analyses, the association between the cardiovascular/ anemia/dementia pattern and the risk of developing ADL was strengthened in women, and that between the sensory impairment/cancer pattern and ADL was strengthened in men, whereas the associations with IADL development were only significant in women. These findings confirm the complexity and heterogeneity of multimorbidity and its consequences for older persons' health status, suggesting the need to go beyond the simple count of chronic conditions and its link with increased risk of functional decline, as previously established [3].

Studies evaluating the topic are few and used different methodologies in analyzing disease clustering. In the Australian Longitudinal Study on Women's Health, where multimorbidity patterns were identified using factor analysis, the authors found that the cardiovascular pattern was associated with the greatest decline in ADL over six years, whereas the neurological/mental health pattern was associated with the greatest decline in IADL, compared to a group of women that did not have multimorbidity at baseline or that were multimorbid but their conditions did not group into any of the identified patterns [12]. Similarly, we compared specific multimorbidity patterns with an unspecific one including diseases that did not exceed the expected prevalence in the total sample.

Several hypotheses can be suggested concerning the differential risk of disability associated with multimorbidity patterns. First, the development of chronic diseases could be an intermediate link among various pathophysiological 
processes happening in older age. In fact, aging-related biological processes such as chronic inflammation and cellular senescence may represent the root mechanisms leading to disability through chronic diseases [13]. In a previous report, we showed that inflammatory marker levels differ according to specific patterns of multimorbidity, being highest in the cardiovascular multimorbidity pattern [11]. Another hypothesis is that the combinations of specific diseases may enhance sarcopenia and the associated disability onset [14], which could be especially relevant in the case of the pattern including musculoskeletal and respiratory diseases, as both conditions are often characterized by loss of lean muscle mass $[15,16]$. One additional possible theory is that combinations of diseases that affect functional ability through different mechanisms may have a worse effect on functional decline than comorbidities sharing common etiologic or pathophysiologic mechanisms [17]. In fact, our findings showed that the pattern including conditions affecting three different organ systems, i.e., cardiac diseases, dementia and anemia, was one of the most burdensome in terms of associated disability risk. Finally, recent evidence suggests that psychiatric disturbances, such as generalized anxiety disorders, are more strongly associated with disability in women than in men [18]. This is in line with our finding whereby the psychiatric pattern was only associated with incident disability in females.

Among the limitations of this study are the fact that the main exposure (i.e., multimorbidity patterns) was only measured at baseline, and the limited transportability of the findings, given that SNAC-K participants are on average wealthier and healthier than the general older population in Sweden. Our strict exclusion criteria is likely to have limited the generalizability of our findings.

\section{Conclusions}

Findings from our study suggest that specific multimorbidity patterns may differentially lead to functional decline, which is important for better understanding the relationship between diseases and disability, for the design of future prevention strategies aimed at delaying functional impairment in old age, and for a better healthcare resource planning.

Supplementary information The online version contains supplementary material available at (https://doi.org/10.1007/s40520-020-01773 $-\mathrm{z})$.

Acknowledgements We thank the SNAC-K participants and the SNAC-K Group for their collaboration in data collection and management.
Authors' Contribution Conception or design of the work was contributed by AM, RA, DLV, ACL. Data analysis was contributed by RA, ARL. Interpretation of the results was contributed by all coauthors. Drafting of the article was contributed by AM, RA, ACL. Critical revision of the manuscript was contributed by all coauthors. Final approval of the manuscript was contributed by all coauthors. All the authors fulfill the ICMJE criteria for authorship.

Funding Open Access funding provided by Karolinska Institute. This work was supported by the funders of the Swedish National study on Aging and Care (SNAC): the Ministry of Health and Social Affairs, Sweden; the participating County Councils and Municipalities; and the Swedish Research Council. Specific grants were received from The Swedish Research Council for Medicine (VR; 521-2013-8676; 2017-06088; 2016-00981); the Swedish Research Council for Health, Working life and Welfare (Forte; 2016-07175; 2017-01764); The Italian Ministry of Health (PE-2016-02364885). The funders had no role in study design, data collection and analysis, decision to publish, or preparation of the manuscript.

\section{Compliance with ethical standards}

Conflict of interest All authors declare that no organizations have attempted to influence the study or the writing of the manuscript. They have had no financial relationships in the previous three years with any organizations that might have an interest in influencing the submitted work and no other relationships or activities that could appear to have influenced the submitted work.

Statement of human and animal rights The present study has been approved by the appropriate ethics committee and has therefore been performed in accordance with the ethical standards laid down in the 1964 Declaration of Helsinki and its later amendments.

Informed consent Written informed consent was obtained from all participants. For persons living in institutions and for people with dementia, a proxy respondent (a next of kin) was also asked for consent in addition to the participant (refer to SNAC-K website for details at https ://www.snac-k.se/).

Data sharing Data are from the SNAC-K project, a population-based study on aging and care (http://www.snac-k.se/). Access to these original data is available to the research community upon approval by the SNAC-K data management and maintenance committee. Applications for accessing these data can be submitted to Maria Wahlberg (Maria. Wahlberg@ki.se) at the Aging Research Center, Karolinska Institutet.

Open Access This article is licensed under a Creative Commons Attribution 4.0 International License, which permits use, sharing, adaptation, distribution and reproduction in any medium or format, as long as you give appropriate credit to the original author(s) and the source, provide a link to the Creative Commons licence, and indicate if changes were made. The images or other third party material in this article are included in the article's Creative Commons licence, unless indicated otherwise in a credit line to the material. If material is not included in the article's Creative Commons licence and your intended use is not permitted by statutory regulation or exceeds the permitted use, you will need to obtain permission directly from the copyright holder. To view a copy of this licence, visit http://creativecommons.org/licenses/by/4.0/. 


\section{References}

1. Calderón-Larrañaga A, Vetrano DL, Onder G, et al (2017) Assessing and measuring chronic multimorbidity in the older population: a proposal for its operationalization. J Gerontol A Biol Sci Med Sci 72:1417-1423

2. Rizzuto D, Melis RJF, Angleman S, et al (2017) Effect of chronic diseases and multimorbidity on survival and functioning in elderly adults. J Am Geriatr Soc 65:1056-1060

3. Marengoni A, von Strauss E, Rizzuto D, et al (2009) The impact of chronic multimorbidity and disability on functional decline and survival in elderly persons. A community-based, longitudinal study. J Intern Med 265:288-295

4. Zhang Y, Zhou L, Liu S, et al (2020) Prevalence, correlates and outcomes of multimorbidity among the middle-aged and elderly: findings from the China Health and Retirement Longitudinal Study. Arch Gerontol Geriatr 90:104135

5. Marengoni A, Angleman S, Fratiglioni L (2011) Prevalence of disability according to multimorbidity and disease clustering: a population-based study. J Comorb 1:11-18

6. Grande G, Marengoni A, Vetrano DL, et al (2021) Multimorbidity burden and dementia risk in older adults: the role of inflammation and genetics. Alzheimers Dement. https://doi.org/10.1002/ alz.12237

7. Vetrano DL, Roso-Llorach A, Fernández S, et al (2020) Twelveyear clinical trajectories of multimorbidity in a population of older adults. Nat Commun 11:3223

8. Quiñones AR, Markwardt S, Botoseneanu A (2016) Multimorbidity combinations and disability in older adults. J Gerontol A Biol Sci Med Sci 71:823-830

9. Quiñones AR, Markwardt S, Thielke S, et al (2018) Prospective disability in different combinations of somatic and mental multimorbidity. J Gerontol A Biol Sci Med Sci 73:204-210

10. Lagergren M, Fratiglioni L, Hallberg I, et al (2004) A longitudinal study integrating population, care and social services data. The
Swedish National study on Aging and Care (SNAC). Aging Clin Exp Res 16:158-168

11. Marengoni A, Roso-Llorach A, Vetrano DL, et al (2020) Patterns of multimorbidity in a population-based cohort of older people: sociodemographic, lifestyle, and functional differences. J Gerontol A Biol Sci Med Sci 75:798-805

12. Jackson CA, Jones M, Tooth L, et al (2015) Multimorbidity patterns are differentially associated with functional ability and decline in a longitudinal cohort of older women. Age Ageing 44:810-816

13. Ferrucci L, Gonzalez-Freire M, Fabbri E, et al (2020) Measuring biological aging in humans: A quest. Aging Cell 19:e13080

14. Kelley GA, Kelley KS, Kelley GA et al (2017) Is sarcopenia associated with an increased risk of all-cause mortality and functional disability? Exp Gerontol 1:100-103

15. Minetto MA, Giannini A, McConnell R, et al (2020) Common musculoskeletal disorders in the elderly: the star triad. J Clin Med 9:1216

16. Roig M, Eng JJ, MacIntyre DL, et al (2011) Deficits in muscle strength, mass, quality, and mobility in people with chronic obstructive pulmonary disease. J Cardiopulm Rehabil Prev 31:120-124

17. Kriegsman DMW, Deeg DJH, Stalman WAB (2004) Comorbidity of somatic chronic diseases and decline in physical functioning: the Longitudinal Aging Study Amsterdam. J Clin Epidemiol 57:55-65

18. Vesga-López O, Schneier FR, et al (2008) Gender differences in generalized anxiety disorder: results from the National Epidemiologic Survey on Alcohol and Related Conditions (NESARC). J Clin Psychiatry 69:1606-1616

Publisher's Note Springer Nature remains neutral with regard to jurisdictional claims in published maps and institutional affiliations. 